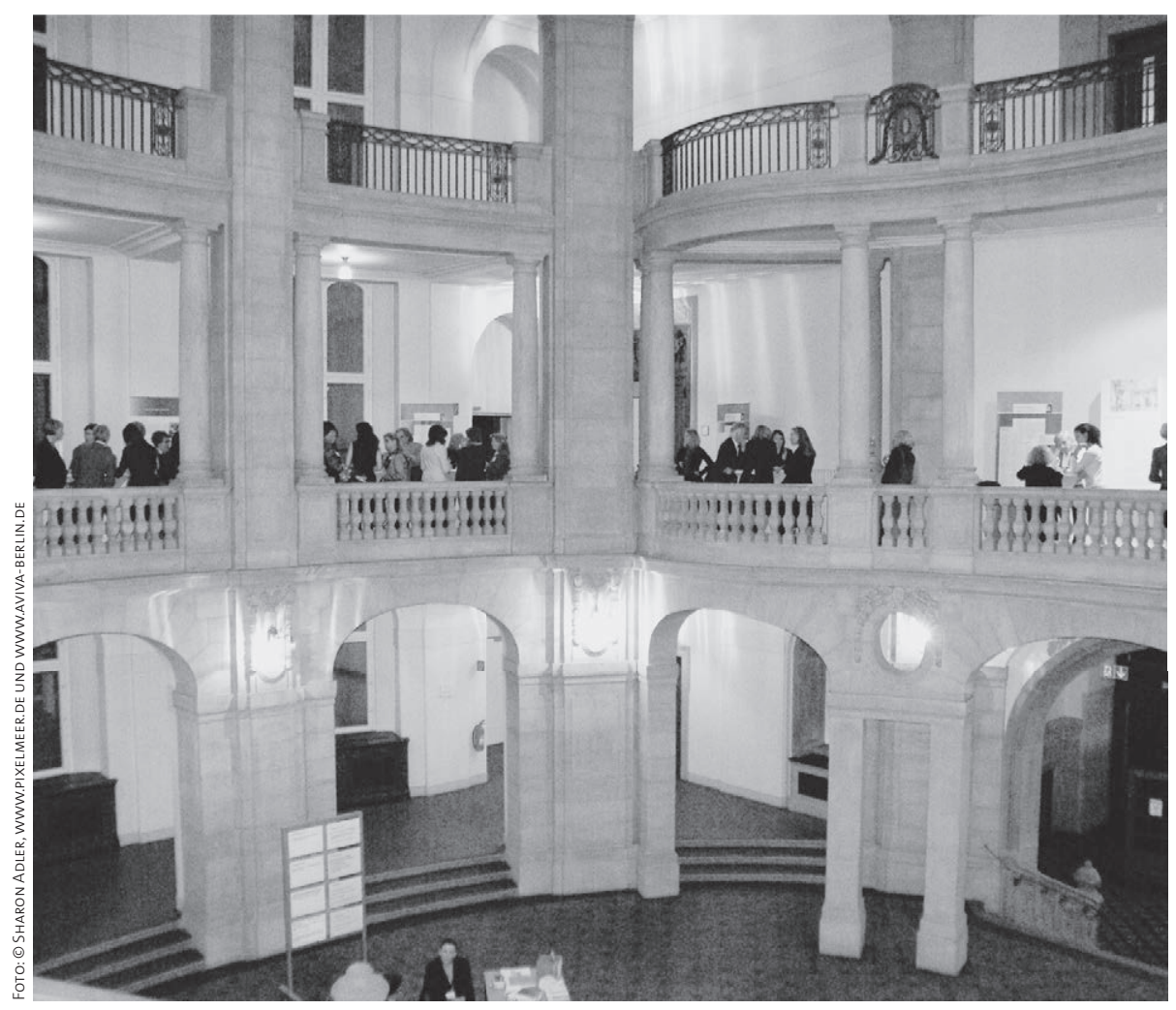

$\checkmark$ Das Kammergericht bietet einen großartigen Rahmen für den Empfang. Im Hintergrund ist die Wanderausstellung des djb „Juristinnen in der DDR“ zu sehen, die 2011 im Landgericht Potsdam eröffnet wurde. Die 23 Ausstellungstafeln befassen sich mit dem Status des Rechts in der DDR, der Rolle der Juristinnen und Juristen im DDRRechtssystem, der juristischen Ausbildung usw. Die weiteren Tafeln porträtieren zwölf Juristinnen aus drei Generationen: (1) der Generation mit bürgerlichem Hintergrund, (2) der DDR-Generation und (3) der Generation mit geteiltem Berufsleben in der DDR und im wiedervereinten Deutschland. Die Ausstellung kann von den Landesverbänden und Regionalgruppen des djb ausgeliehen werden, aber auch von anderen geeigneten öffentlichen und privaten Institutionen wie Gerichten und Ministerien, die sich mit dem Thema Juristinnen und DDR auseinandersetzen möchten. Sie besteht aus 23 farbigen Roll-ups $(215 \times 85 \mathrm{~cm})$ und kann ohne großen technischen Aufwand präsentiert werden.

\title{
Sitzung des Regionalgruppenbeirates vom 20. bis 22. April 2012 in Bremerhaven
}

\section{Andrea Kirberger \\ Vorstand Regionalgruppenbeirat, Stellvertretende Vorsitzende der RG Dresden; Rechtsanwältin, Fachanwältin für Bau- und Ar- chitektenrecht, Dresden}

Der Regionalgruppenbeirat (RGB) des djb tagt einmal jährlich mit dem Ziel des Informations- und Erfahrungsaustausches zwischen den Regionalgruppen und Landesverbänden. In diesem Jahr kamen vom 20. bis 22. April 2012 insgesamt 22 Delegierte aus ganz Deutschland in Bremerhaven zusammen. Dabei scheuten die Teilnehmerinnen keinen zeitlichen Aufwand, sogar aus Konstanz und aus Brüssel waren Vertreterinnen angereist.

Zum Auftakt der Sitzung am Samstagvormittag ließ es sich der Präsident des Amtsgericht Bremerhaven, Uwe Lissau, der freundlicherweise einen Sitzungssaal im Amtsgericht für die Tagung zur Verfügung gestellt hatte, nicht nehmen, ein Grußwort an die angereisten Teilnehmerinnen zu richten.

Anschließend berichtete die Präsidentin des djb, Ramona Pisal, über die Arbeit des djb auf Bundesebene und insbesondere ihre Tätigkeit seit dem Bundeskongress in Potsdam, auf dem sie bei der Mitgliederversammlung zur neuen Präsidentin gewählt worden war. Die Teilnehmerinnen waren beeindruckt von der Vielzahl der wahrgenommenen Termine und geknüpf- ten bzw. aufgefrischten Kontakte. Mit großem Interesse sind auch die Ausführungen von Ramona Pisal zur Aktion „Frauen in die Roten Roben“, die von der Vizepräsidentin des djb Eva Schübel initiiert worden ist, sowie zu dem nunmehr schon im dritten Jahr laufenden Projekt „Aktionärinnen fordern Gleichberechtigung “ aufgenommen worden. Ramona Pisal verwies ergänzend auf die „Berliner Erklärung“ mit der Bitte, diese unter www.berlinererklaerung.de zu unterstützen.

Bundesgeschäftsführerin Anke Gimbal gab einen Überblick über die geplanten Veranstaltungen, so insbesondere den djbEmpfang beim Deutschen Juristentag am 19. September 2012 sowie den nächsten Bundeskongress in Leipzig im September 2013. Sie erinnerte darüber hinaus noch einmal an die im Rahmen des Kongresses in Potsdam im vergangenen Jahr eröffnete Wanderausstellung „Juristinnen in der DDR “, die von den Regionalgruppen und Landesverbänden präsentiert werden kann.

Anne Brozat, Vertreterin der Jungen Juristinnen im Bundesvorstand, erläuterte das von ihr und ihrer Kollegin Katharina König entwickelte Mentoring-Programm, in dessen Rahmen von Ursula Matthiessen-Kreuder Telefonkonferenzen zum Thema Karriereplanung und Berufsorientierung geleitet werden. Katharina König und Anne Brozat haben darüber hinaus bereits konkrete Ideen, wie das Mentoring-Programm ausgeweitet werden kann. 
Im Anschluss an die Berichte der angereisten Bundesvorstandsmitglieder hielt Dagmar Freudenberg, Vorsitzende der Strafrechtskommission des djb, einen hochkarätigen und äußerst interessanten Vortrag zum Thema „10 Jahre Gewaltschutzgesetz“. Einleitend berichtete Dagmar Freudenberg über die Arbeit der Strafrechtskommission, um dann die historische Entwicklung des Themas häusliche Gewalt und die diesbezügliche Arbeit des djb zu erläutern. Das Gewaltschutzgesetz aus dem Jahr 2002, welches sowohl zivilrechtliche als auch strafrechtliche und polizeirechtliche Regelungen beinhaltet, wurde so für viele Teilnehmerinnen erstmals transparent und hinsichtlich seiner praktischen Anwendung nachvollziehbar. Abschließend wies Dagmar Freudenberg auf das geplante Seminar zum Thema „10 Jahre Gewaltschutzgesetz“ am 26. und 27. Oktober 2012 in Bonn hin.

Der Nachmittag des 21. April 2012 war dem Erfahrungsund Ideenaustausch zwischen den Regionalgruppen vorbehalten. Die Vertreterinnen der Regionalgruppen erzählten jeweils ausführlich von ihrer Arbeit im vergangenen Jahr, die durchgeführten Veranstaltungen sowie die Entwicklung der Mitgliederzahlen und Mitgliederbeteiligung. In teilweise lebhaften Diskussionen wurden Anregungen für zukünftige Veranstaltungen sowie für die Mitgliedergewinnung gegeben.

Am Sonntagvormittag berichtete der Vorstand des Regionalgruppenbeirates über seine Tätigkeit, zum Beispiel den Besuch der Bundesvorstandssitzungen, und wies auf einige Aspekte betreffend die Regionalgruppen hin. Wichtig erscheint, dass die Homepages der Regionalgruppen und Landesverbände möglichst aktuell sind. Sollten technische Probleme auftreten, können diese an den Vorstand des Regionalgruppenbeirates oder die Geschäftsstelle herangetragen werden. Noch einmal erläutert wurde, dass eine Mailingliste für den

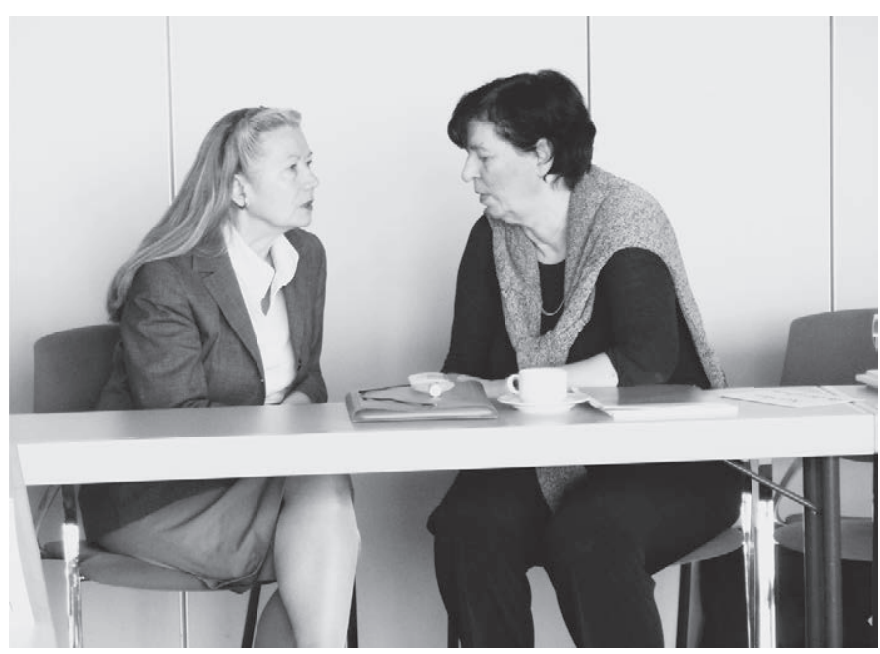

$\Delta$ Ramona Pisal (links), Präsidentin des djb, im Gespräch mit Rechtsanwältin Birgit Kersten, Vorstand RGB und Projektmitarbeiterin, u.a. über die weitere Planung des Projektes „Aktionärinnen fordern Gleichberechtigung“. Bei dem vom BMFSFJ von August 2009 bis April 2014 geförderten Projekt besuchen Vertreterinnen des djb pro Jahr jeweils etwa 75 Hauptversammlungen und richten kritische Fragen an den Vorstand und den Aufsichtsrat zum Thema Frauen in Führungspositionen. (Foto: Andrea Kirberger, Vorstand RGB, Dresden)

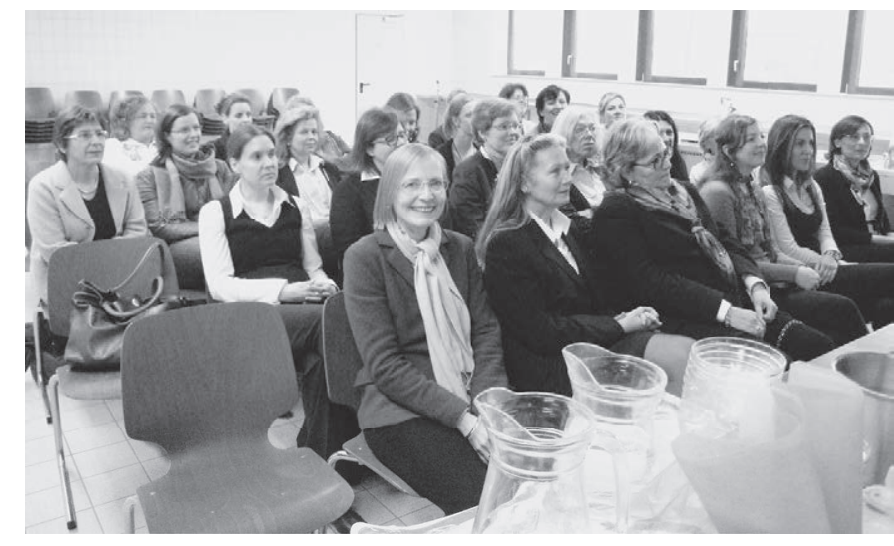

A Den Samstagabend verbrachten die Teilnehmerinnen der RGB-Sitzung bei einer Kochshow im 1927 als Seefischlehrküche gegründeten Bremerhavener Seefischkochstudio. Die Kochshow bot unter Anleitung von Koch Murat Kirhan „eine kulinarische Welt des Seefisches mit Tipps und Tricks zum Dünsten, Kochen, Braten und Backen“. Auch Vorschläge für ein kaltes Buffet aus Neptuns Reich fehlten nicht. Das Event wurde abgeschlossen mit dem versprochenen „hemmungslosen Fischkonsum“ der live zubereiteten Gerichte und einem kalt-warmen Fischbuffet zum Sattessen. (Foto: Christina Lorenz, Vorsitzende LV Thüringen)

Regionalgruppenbeirat, in der als Empfängerinnen die Vorsitzenden aller Regionalgruppen und Landesverbände sowie von diesen jeweils benannte Delegierte eingetragen sind, existiert. Die Mailingliste kann auch von den Regionalgruppen genutzt werden, wenn Informationen an alle Regionalgruppen und Landesverbände weitergegeben werden sollen.

Nach wie vor auf Interesse stößt die Aktion „BuVo transparent“, bei der Regionalgruppenvertreterinnen die Möglichkeit geboten wird, an Bundesvorstandssitzungen teilzunehmen. Für die nächsten Bundesvorstandssitzungen sind die Regionalgruppe Hannover und der Landesverband Hamburg sowie daran anschließend gemäß neuer Auslosung der Landesverband Hessen, die Regionalgruppe München/Südbayern und die Regionalgruppe Köln vorgesehen.

Neben der äußerst informativen Tagung bot sich die Möglichkeit, Bremerhaven durch eine zweistündige, hochinteressante Rundfahrt mit dem Hafenbus besser kennenzulernen. Außerdem nutzten viele Teilnehmerinnen die Gelegenheit, das Deutsche Auswandererhaus zu besichtigen. Am Samstagabend durfte selbstverständlich das Seefischkochstudio nicht fehlen, nachdem bereits am Freitagabend die Möglichkeit zu einem geselligen Beisammensein und Kennenlernen im Ratskeller bestanden hatte, zu dem sogar der Stadtverordnetenvorsteher Artur Beneken gekommen war, um die Teilnehmerinnen in Bremerhaven zu begrüßen.

Insgesamt war die Tagung in Bremerhaven eine Bereicherung und hat den Kontakt zwischen den einzelnen Regionalgruppen und Landesverbänden (wieder) verstärkt. Besonderer Dank für die Vorbereitung der großartigen Veranstaltung gilt Birgitt Nussbaum und Roswitha Seidel sowie Birgit Kersten, die sich in besonderem Maß dafür eingesetzt haben, dass der Aufenthalt in Bremerhaven sicherlich noch allen Teilnehmerinnen lange in positiver Erinnerung bleiben wird. 